\title{
Mismatch and synchronization: Influence of asymmetries in systems of two delay-coupled lasers
}

\author{
K. Hicke, ${ }^{1,2}$ O. D’Huys, ${ }^{3}$ V. Flunkert, ${ }^{2}$ E. Schöll, ${ }^{2}$ J. Danckaert, ${ }^{3}$ and I. Fischer ${ }^{1}$ \\ ${ }^{1}$ Instituto de Fisica Interdisciplinar y Sistemas Complejos (IFISC), UIB-CSIC, Campus Universitat de les Illes Balears, \\ E-07122 Palma de Mallorca, Spain \\ ${ }^{2}$ Institut für Theoretische Physik, TU Berlin, Hardenbergstraße 36, D-10623 Berlin, Germany \\ ${ }^{3}$ Applied Physics Research Group (APHY), Vrije Universiteit Brussel, Pleinlaan 2, B-1050 Brussel, Belgium
}

(Received 29 December 2010; published 18 May 2011)

\begin{abstract}
We study the synchronization properties of the delay dynamics of two identical semiconductor lasers coupled through a semitransparent mirror. Via an analytical and numerical approach, we investigate the influence of asymmetries, in particular mismatches of self- and cross-coupling strength and differences in self- and crosscoupling delay. We show that the former mismatch affects the stability of the zero-lag state but not the dynamics within the synchronization manifold, while the latter mismatch does not affect the quality of synchronization but alters the dynamics significantly. Our results are extended to different unidirectional coupling schemes. This is highly relevant for communication schemes utilizing chaotic dynamics. Finally, the influence of nonlinear gain saturation on the dynamics and stability of synchronization is discussed.
\end{abstract}

DOI: 10.1103/PhysRevE.83.056211

PACS number(s): 89.75.Kd, 05.45.Xt, 89.75.Hc, 02.30.Ks

\section{INTRODUCTION}

Synchronization of coupled nonlinear systems has been a focus of research over the recent decades in different fields such as neuroscience, biology, chemistry, and social sciences [1-3]. In many technological and biological applications, synchronizing individual elements of an extended system is necessary for coherent operation, e.g., in the brain, where the synchronization of neurons is believed to play an important role, for instance, in the binding problem [4,5].

For technological applications, a particularly interesting effect is chaos synchronization [6-8], since it may provide new secure communication schemes [9]. In this context, semiconductor lasers have been proposed [10-13] as a physical realization of chaos-based cryptography systems, because this would allow the use of existing communication infrastructures [14].

Coupling lasers over finite distances introduces a coupling delay due to the limited signal propagation speed. Mathematically, such delay terms render the system infinitely dimensional and result in rich dynamical behavior including hyperchaos $[15,16]$. In addition, coupling delays affect the synchronization properties [17-20].

For instance, it has been shown that in a setup of bidirectionally coupled lasers zero-lag chaos synchronization is impossible if the coupling delay is longer than the intrinsic dynamical time scale [21]. For lasers, this corresponds with coupling distances larger than a few meters. In the case of mutually coupled lasers, the zero-lag chaos synchronization exists but is unstable [22]. Recently, these results were generalized $[19,20]$ to assess the synchronizability of any network with a large coupling delay and an arbitrary type of nodes. In particular, it was shown that chaos synchronization is generally unstable in the network motif of two bidirectionally coupled systems. Instead, there occurs a more generalized synchronization dynamics of the leader-laggard type. In other words, the lasers do not synchronize identically, but with a constant time lag equal to the coupling delay time [20,23]. Under fully symmetric conditions, the role of the leader and laggard is defined by the initial conditions and may switch chaotically [24-26].

Recently, it has been shown that a relay component, such as a semitransparent mirror [27,28] or a third laser [29-31] in the middle of the two lasers, can stabilize zero-lag synchronization. Similar effects might even occur in the brain, where relay populations can help synchronize two other spatially distant neuron populations [32-34].

Besides bidirectionally coupled lasers, other coupling schemes have been considered for chaos synchronization and communication. The most common scheme is the transmitterreceiver setup, where a transmitter laser is unidirectionally coupled to a receiver laser [16,35-37]. Here, different configurations such as open- and closed-loop setups have been considered (see Sec. III).

From a nonlinear dynamics perspective, in all of the above coupling schemes, chaos synchronization is stable if the chaotic attractor in the synchronization manifold is transversely stable, i.e., the maximum transverse Lyapunov exponent is negative. It has been recognized that the transverse stability of periodic orbits embedded in the attractor is the key to the transverse stability properties of the attractor itself [38]. This is particularly true if noise is present in the system, such as spontaneous emission noise in lasers. Then, even if the maximum transverse Lyapunov exponent is negative, noise may induce desynchronization close to transversely unstable periodic orbits in the attractor. This behavior has been coined bubbling [39] and has recently been found for delay-coupled lasers [40]. In lasers with self-feedback or in coupled laser systems, the fundamental periodic orbits which structure the phase space are the external cavity modes (ECMs) [41,42].

In this work we aim to understand the chaos synchronization properties of different coupling schemes of two lasers by analytically and numerically investigating the modes arising from the particular setup and the stability properties of these modes. Our aim is to understand the influence of asymmetries, in particular parameter and delay mismatches, on the synchronization quality in these setups. This is of general importance, especially for experiments where unavoidable mismatches always lead to asymmetries. 
The paper is organized as follows. In Sec. II, we study a model of two lasers bidirectionally coupled via a semitransparent mirror and address the effect of a mismatch in the reflection and transmission of the relay mirror. We show that bubbling is enhanced as the mismatch is increased. In Sec. III, we compare the synchronization dynamics of a bidirectional configuration with a relay and a drive-response setup. We find that the stability of synchronization for the open- and closed-loop configuration corresponds to the synchronization stability for a bidirectional setup with symmetrical and asymmetrical coupling, respectively. In Sec. IV, we consider a configuration where the mirror is no longer in the exact middle of the two lasers, i.e., introducing a delay mismatch term to the model. We show that, in this case, the lasers still synchronize identically, but with a time lag corresponding to the delay mismatch. Moreover, the spectrum of ECMs, and thus the dynamics of the systems, is altered significantly due to the delay mismatch. In Sec. V, we focus on the influence of nonlinear gain saturation in the Lang-Kobayashi model on the dynamics and the stability of synchronization. Finally, we draw conclusions in Sec. VI. Details of the stability calculations of the ECMs are shown in the Appendix.

\section{RELAY CONFIGURATION WITH COUPLING MISMATCH}

As a first configuration, we consider two identical semiconductor lasers bidirectionally coupled to each other with a coupling strength $K$. In addition, they each receive selffeedback with a strength $L$. Both the coupling and the feedback are delayed. This can physically be realized through a semitransparent mirror placed between the lasers, with reflectivity $L$ and transmission $K$. This system can then be described by

$$
\begin{aligned}
& \dot{X}_{1}=f\left(X_{1}\right)+L C X_{1}(t-\tau)+K C X_{2}(t-\tau), \\
& \dot{X}_{2}=f\left(X_{2}\right)+K C X_{1}(t-\tau)+L C X_{2}(t-\tau),
\end{aligned}
$$

with $X_{j}=\left(E_{j}, n_{j}\right)$, where the dimensionless variables $E_{j}$ and $n_{j}$ represent the complex electric field amplitude and the excess carrier density of the $j$ th laser, respectively; $f\left(X_{j}\right)$ represents the nonlinear laser dynamics according to the Lang-Kobayashi equations [43]; and

$$
C=\left(\begin{array}{ll}
1 & 0 \\
0 & 0
\end{array}\right)
$$

is a coupling matrix describing the optical feedback and the optical coupling between the lasers (i.e., the optical fields are coupled). The coupling strengths $K=\kappa e^{i \phi_{K}}$ and $L=$ $\lambda e^{i \phi_{L}}$ are the complex self-feedback and coupling amplitudes, respectively. For simplicity, we choose identical feedback and coupling delay $\tau$ (see Sec. IV for the case of unequal delays), and identical feedback and coupling phase $\phi_{L}=\phi_{K}=0$. A scheme of this configuration is depicted in Fig. 1. The nonlinear laser dynamics is described via

$$
f\left(\begin{array}{c}
E_{j} \\
n_{j}
\end{array}\right)=\left(\begin{array}{c}
\frac{1}{2}(1+i \alpha)\left[G\left(n_{j}, E_{j}\right)-1\right] E_{j}+F_{E} \\
\frac{1}{T}\left[p-n_{j}-G\left(n_{j}, E_{j}\right)\left|E_{j}\right|^{2}\right]
\end{array}\right),
$$

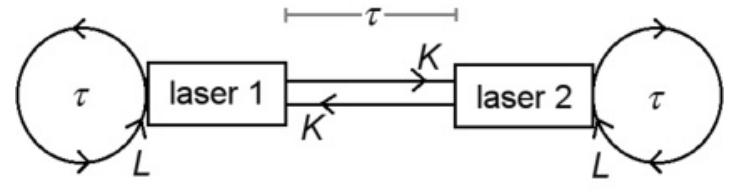

FIG. 1. Symmetric setup of bidirectionally coupled lasers with feedback. The distances are given in time for a one-way trip. $L$ and $K$ are the strengths of the feedback and the coupling, respectively.

with the gain function

$$
G\left(n_{j}, E_{j}\right)=\frac{n_{j}+1}{1+\mu\left|E_{j}\right|^{2}} .
$$

Here, $\alpha$ is the linewidth enhancement factor, $p$ is the pump current in excess of the laser threshold, $\mu$ models the nonlinear gain saturation, and the time scale parameter $T$ is the ratio of the carrier and the photon lifetimes; all parameters are dimensionless in this model. The field noise $F_{E}$ originating from spontaneous emission is modeled as Gaussian white noise with correlation $\left\langle F_{E}(s) \overline{F_{E}\left(s^{\prime}\right)}\right\rangle=\beta\left(n+n_{0}\right) \delta\left(s-s^{\prime}\right)$ and zero mean $\left\langle F_{E}(s)\right\rangle=0$. Here, $n_{0}=10$ is the carrier density at the threshold in dimensionless units and $\beta=10^{-5}$ is the spontaneous emission factor.

Without self-feedback $(L=0)$, the two lasers are coupled face to face. As discussed in Sec. I, it is known that the identically synchronized solution is unstable in such a configuration, and the lasers are in a generalized synchronization of the leader-laggard type [23,24]. For $K=0$, we have two separate, identical chaotic systems. If the coupling is symmetric in strength and in phase $(L=K)$, then the zero-lag synchronized solution can be stabilized [27], but bubbling is still present in the system [40].

To analyze the stability of this zero-lag synchronization, we define a symmetric variable $S(t)=\frac{1}{2}\left[X_{1}(t)+X_{2}(t)\right]$ and an antisymmetric variable $A(t)=\frac{1}{2}\left[X_{1}(t)-X_{2}(t)\right]$. The dynamics in the new variables is described by

$$
\begin{aligned}
& \dot{S}=\frac{1}{2}[f(S+A)+f(S-A)]+(L+K) C S(t-\tau), \\
& \dot{A}=\frac{1}{2}[f(S+A)-f(S-A)]+(L-K) C A(t-\tau) .
\end{aligned}
$$

The identically synchronized state $A(t)=0$ always exists, independent of the feedback strength $L$ and the coupling strength $K$, due to the remaining symmetry of the system. When synchronized, the system behaves like one laser with a feedback equal to the sum of self-coupling and cross coupling $K+L$, and does not depend, in particular, on the mismatch $L-K$. The linear stability of this zero-lag state, however, is given by the time-dependent variational equation for a small transverse perturbation $\delta A(t)$,

$$
\dot{\delta A}(t)=D f(S(t)) \delta A(t)+(L-K) C \delta A(t-\tau),
$$

and does depend on the mismatch $L-K$ between selfcoupling and cross coupling. Here, $D f(S(t))$ denotes the Jacobian of $f$ evaluated on the trajectory $S(t)$. Note that for symmetric coupling $(L=K)$, the explicit delay dependence vanishes.

We calculate numerically the cross-correlation $C(\Delta t)=$ $\sum_{s=-\infty}^{\infty} I_{1}(s) I_{2}(\Delta t+s)$, where $I_{j}=\left|E_{j}\right|^{2}$ is the optical intensity, at zero lag for varying self- and cross-coupling strengths, 


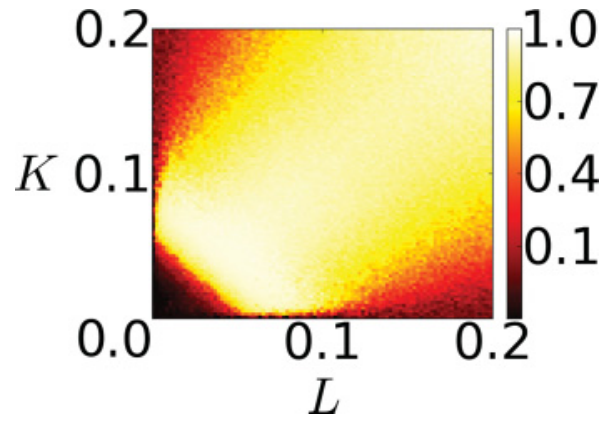

FIG. 2. (Color online) Zero-lag cross correlation $C(0)$ of the two coupled lasers' intensities vs the coupling strength $K$ and feedback strength $L$. Here, the coupling and the feedback have the same delay. Parameters: $\mu=0.26, p=1.0, \alpha=4.0, T=200, \tau=1000$, and noise $\beta=10^{-5}$.

and we obtain an $L=K$ axially symmetric correlation plot (Fig. 2). Large $C(0)$ corresponds to good synchronization. The level of synchronization depends only on the absolute value $|L-K|$ of the coupling mismatch and not on the sign. This symmetry occurs for large delay (as is the case here) and is well understood $[19,20]$.

The chaotic dynamics of a laser with delayed feedback is mainly organized by the external cavity modes (ECMs) $[42,44]$. The ECMs are rotating wave solutions of the form $E(t)=A e^{i \omega t}$ and $n(t)=n$, with constant amplitude $A$, frequency $\omega$, and carrier density $n$ of the lasers. These modes lie on an ellipse in the $(\omega, n)$ plane. On the lower half of the ellipse, the solutions result from constructive interference in the external cavity. These solutions are foci and are called modes. The solutions on the upper half of the ellipse, however, result from destructive interference, and are saddle points. They are called antimodes.

A well-studied dynamical regime of a semiconductor laser with feedback is the low frequency fluctuation (LFF) regime. In the LFF regime [44,45], the laser switches chaotically between the modes, with a drift toward the maximum gain mode located at the bottom of the ellipse. During this process, intensity builds up until the trajectory is ejected along the unstable manifold of a saddle point causing a power dropout.

For two coupled lasers, it has been shown that the desynchronization dynamics can be related to the ECMs in the synchronization manifold $[40,46]$. Starting from Eq. (8), it is possible to calculate the transverse stability of the ECMs. This is discussed in detail in the Appendix. In the neighborhood of transversely stable modes, the two lasers synchronize well, while bubbling typically occurs in the neighborhood of the transversely unstable modes [40].

Without mismatch between self-coupling and crosscoupling $(L=K)$, the modes involved in the dynamics are transversely stable, and the antimodes are transversely unstable. Consequently, the lasers only desynchronize during the power dropout, as shown in Fig. 3(a). This is because only during power dropouts, which result in an excursion of the dynamics toward the solitary laser mode, does the trajectory approach an unstable antimode and desychronization can occur. With increasing mismatch $L-K$, while keeping $K+L$ constant and thus leaving the synchronized dynamics unchanged, an increasing number of modes involved in the

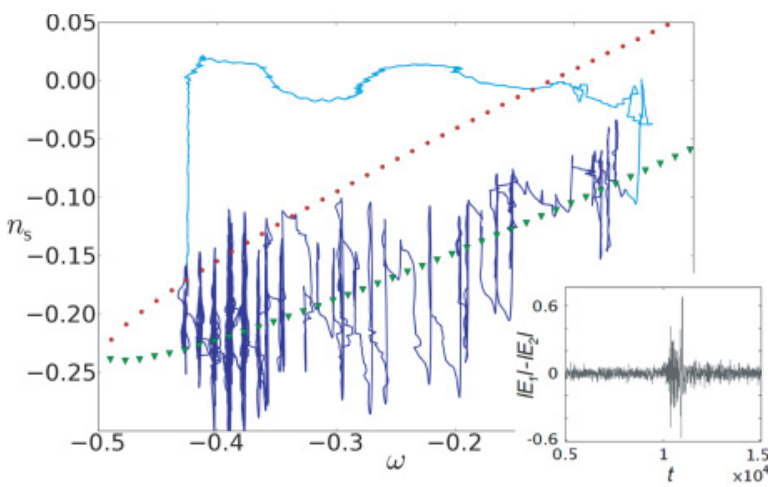

(a)

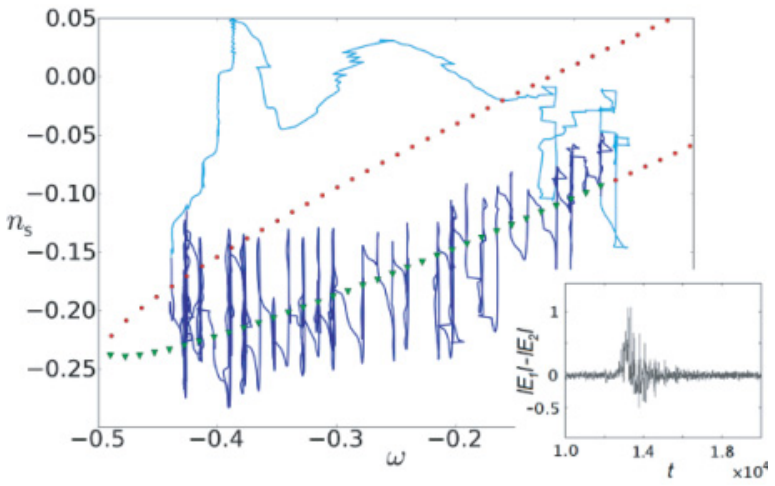

(b)

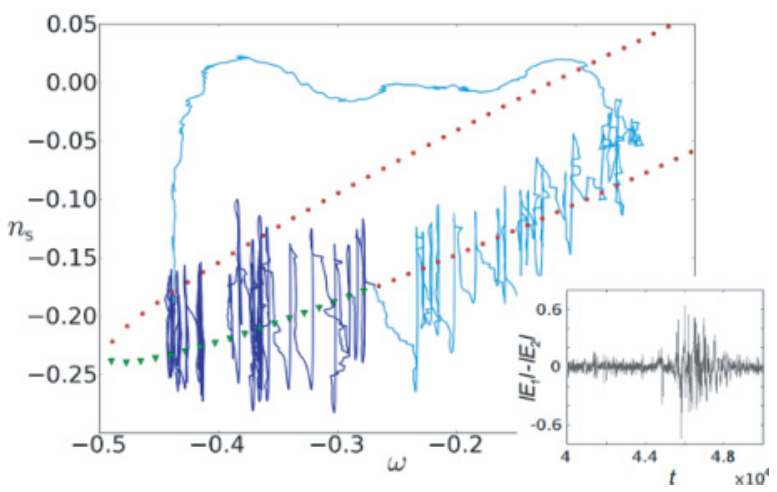

(c)

FIG. 3. (Color online) Dynamics of the symmetrized solution $\left(E_{S}, n_{S}\right)$ of two lasers coupled through a semitransparent mirror with different reflectivity-transmission mismatches, plotted in $\left(\omega, n_{S}\right)$ phase space. $\omega=\left[\phi_{S}(t)-\phi_{S}(t-\tau)\right] / \tau$ is the delay-averaged frequency. Here the symmetrized variable $\phi_{S}=\frac{1}{2}\left(\phi_{1}+\phi_{2}\right)$ describes the phase in the synchronization manifold, $n_{S}=\frac{1}{2}\left(n_{1}+n_{2}\right)$ is the corresponding carrier density, and $E_{S}$ is the field amplitude. The dark blue (black) part of the time trace is well synchronized, the light blue (light gray) part is not. Transversely stable modes are (green) triangles and unstable modes are (red) circles. The insets show the corresponding intensity difference. Parameters: $\tau=500, T=200$, $\alpha=4, p=0.1, \beta=10^{-5}$, and (a) $K=L=0.06$, (b) $K=0.045$, $L=0.075$, and (c) $K=0.03, L=0.09$.

buildup process become transversely unstable. We therefore observe desynchronization not only during power dropouts, but also during the power buildup process [Figs. 3(b) and $3(c)]$. As mentioned above, the synchronized dynamics itself only depends on the sum $K+L$ and thus remains qualitatively unchanged (Fig. 3). 
For $K=0$ or $L=0$, the transversal and longitudinal (within the synchronization manifold) stability properties of the modes are equal, and since all modes, except for the maximum gain mode, are longitudinally unstable, zero-lag synchronization is deterministically unstable too [22]. The correlation is thus small (see Fig. 2).

In the coherence collapse regime, which occurs for higher pump currents, the dynamics can be described as a chaotic itinerancy between modes and antimodes. Also in this case, the number of transversely stable modes decreases with increasing mismatch, leading to more desynchronization events. We also note that the number of stable modes decreases for increasing pump current if the gain saturation effect is not accounted for, i.e., $\mu=0$. The transverse stability of the modes is higher, taking into account gain saturation, which explains the broader area of high synchronization levels in Fig. 2. For a more detailed discussion of the nonlinear gain, see Sec. V.

The correlation between the two lasers at zero lag depends on the difference of self-coupling and cross coupling, but it does not depend on the sign of the mismatch, i.e, whether $K>$ $L$ or $L>K$. This can again be understood by looking at the ECMs' transverse stability, which depends on the magnitude of $L-K$, but not on the sign.

When investigating the correlation at nonzero lags and the stability of synchronization of the leader-laggard type, we get different results. Considering the peaks of the crosscorrelation function at shifts of multiples of the delay time, $k \tau, k \neq 0$ [Fig. 4(b)], we find that if $L-K$ increases (positive mismatch), all considered non-zero-lag peaks decrease in the same way. The corresponding peaks in the autocorrelation and thus the dynamics of the lasers do not change much with a positive mismatch [Fig. 4(a)].

For a negative mismatch $(K>L)$, the lasers evolve gradually from identical zero-lag synchronization to generalized synchronization of the leader-laggard type: the crosscorrelation peak at one delay time and other odd numbered peaks do not change much with the mismatch, but the correlation at zero lag and at even multiples of the delay vanish [Fig. 4(b)]. Also the autocorrelation is affected: the even peaks remain and the odd peaks vanish [Fig. 4(a)]. Note that the sum of the autocorrelation and cross-correlation function is symmetric for positive and negative mismatch (not shown).

\section{DRIVE-RESPONSE CONFIGURATION: OPEN AND CLOSED LOOP}

A coupling configuration that is frequently used for chaos communication purposes is a drive-response configuration $[10,14,47,48]$. The drive laser, or transmitter, is subject to its own delayed feedback; the responding laser, or receiver, receives a chaotic input from the transmitter. In a so-called closed-loop configuration, which is depicted schematically in Fig. 5, the receiver is also a chaotic element subject to its own feedback. In the open-loop configuration, the receiver has no self-feedback and therefore exhibits a stable continuous wave output when decoupled from the transmitter. Both cases can be modeled by

$$
\begin{gathered}
\dot{X}_{1}=f\left(X_{1}\right)+K C X_{1}(t-\tau), \\
\dot{X}_{2}=f\left(X_{2}\right)+(1-\epsilon) K C X_{1}\left(t-\tau_{c}\right)+\epsilon K C X_{2}(t-\tau) .
\end{gathered}
$$

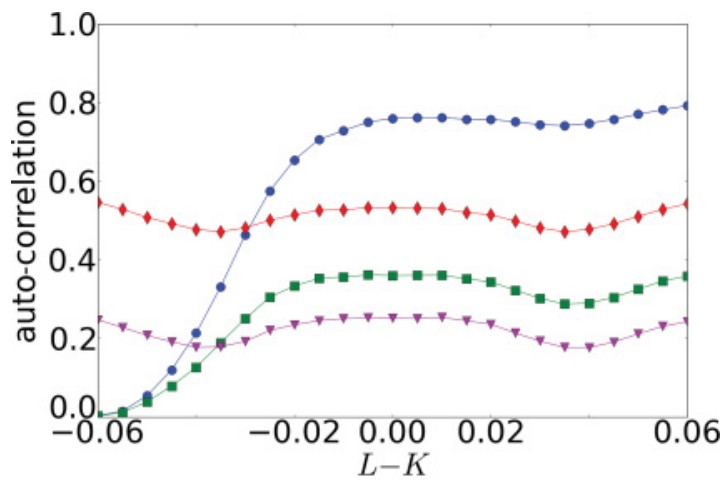

(a)

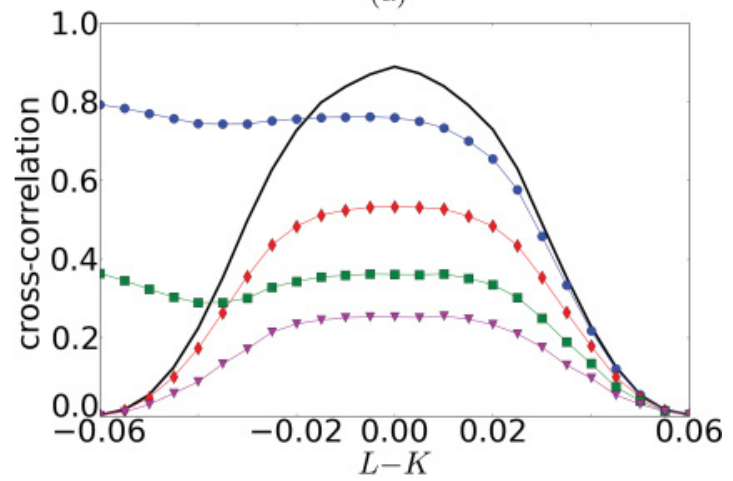

(b)

FIG. 4. (Color online) Evolution of the peaks of the autocorrelation and cross-correlation function $C(\Delta t)$, respectively, with the mismatch $L-K$. Both correlations are calculated for the lasers' intensities: (a) autocorrelation and (b) cross-correlation. Black thick line: cross-correlation at zero lag $C(0)$. Blue circles: peak at one delay time $C(\tau)$. Red diamonds: $C(2 \tau)$. Green squares: $C(3 \tau)$. Magenta triangles: $C(4 \tau)$. Parameters: $\tau=1000, \mu=0, p=1, T=200$, $\beta=10^{-5}$.

If $\epsilon=0$, we have an open-loop receiver, and if $\epsilon>0$, the configuration is a closed loop.

The dynamics in the synchronization manifold is the dynamics of the transmitter laser, $S(t)=X_{1}(t)$. Synchronization without any time shift occurs only for $\tau_{c}=\tau$. If $\tau>\tau_{c}$, the receiver laser's dynamics anticipates the transmitter's dynamics, and for $\tau<\tau_{c}$, the receiver lags behind the transmitter. However, we note that the coupling delay $\tau_{c}$ does not affect the dynamics in a unidirectional coupling configuration. The time shift between the dynamics of both lasers corresponds to $\tau-\tau_{c}$. The antisymmetric direction can thus be defined as $A(t)=X_{1}(t)-X_{2}\left[t-\left(\tau-\tau_{c}\right)\right]$. In terms of the new variables, the dynamics can be rewritten as

$$
\begin{gathered}
\dot{S}=f(S)+K C S(t-\tau), \\
\dot{A}=f(S)-f(S-A)+\epsilon K C A(t-\tau) .
\end{gathered}
$$

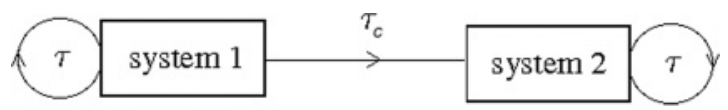

FIG. 5. Scheme for a closed-loop setup as a drive-response configuration. The feedback delay is $\tau$. 


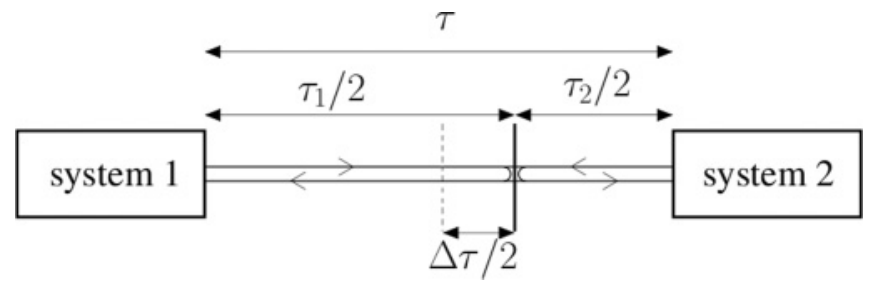

FIG. 6. Scheme of a bidirectional coupling setup with a delay mismatch of the self-feedback. The distances are given in time for a one-way trip. Both self-feedback strengths have the value $L$ and the coupling strengths both equal $K$.

When linearizing around $A(t)=0$, we find that the linear stability of the zero-lag synchronized solution in an openloop receiver $(\epsilon=0)$ is the same as the setup of two lasers coupled through a semitransparent mirror, without self- and cross-coupling mismatch $(L=K)$. In contrast, in a closedloop receiver, the stability of the synchronization manifold is the same as in a mirror setup with a mismatch between self-coupling and cross-coupling.

In chaotic communication, the open-loop configuration is traditionally preferred over the closed-loop scheme because it is more robust against parameter mismatches and much easier to implement. In addition, the resynchronization time in the case of a sudden interruption of the connection is much shorter (see Ref. [13] and references therein). It was shown [37], however, that if the feedback delays are identical, the synchronization quality for the closed-loop rather than for the open-loop scheme is much higher. Also Ref. [49] showed that the performance of closed-loop receivers are less sensible to detuning between the emitter and receiver. Furthermore, several methods considering chaotic communications have been proposed that take advantage of specific properties of the closed-loop configuration $[13,50]$.

\section{RELAY CONFIGURATION WITH DELAY MISMATCH}

\section{A. Synchronization properties}

We come back to the case of bidirectionally coupled lasers with self-feedback. As discussed above, this coupling scheme can be realized experimentally with a semitransparent mirror between the lasers. We now assume that the mirror is no longer positioned in the perfect middle between the two lasers. The lasers are hence subject to a different feedback delay $\tau_{1,2}=$ $\tau \pm \Delta \tau$, but they still experience the same coupling delay $\tau=\frac{1}{2}\left(\tau_{1}+\tau_{2}\right)$, since the distance between the lasers does not change. A schematic representation of the setup is shown in Fig. 6.

We can then model this system via the equations

$$
\begin{aligned}
\dot{X}_{1}= & f\left(X_{1}\right)+\frac{1}{2} L C X_{1}[t-(\tau+\Delta \tau)] \\
& +\frac{1}{2} K C X_{2}(t-\tau), \\
\dot{X}_{2}= & f\left(X_{2}\right)+\frac{1}{2} L C X_{2}[t-(\tau-\Delta \tau)] \\
& +\frac{1}{2} K C X_{1}(t-\tau),
\end{aligned}
$$

where we consider, without loss of generality, $\Delta \tau \geqslant 0$.

The first laser then has a larger self-feedback delay $\left(\tau_{1}=\tau+\Delta \tau\right)$ than the second laser $\left(\tau_{2}=\tau-\Delta \tau\right)$. Zerolag synchronization $X_{1}(t)=X_{2}(t)$ is no longer a solution of this system. Instead a time-shifted synchronized solution $X_{1}(t)=X_{2}(t-\Delta \tau)$ is possible [31]. To analyze the stability of this time-shifted identically synchronized solution, we define the symmetric variable as $S(t)=\frac{1}{2}\left[X_{1}(t+\Delta \tau)+\right.$ $\left.X_{2}(t)\right]$ and the antisymmetric variable as $A(t)=\frac{1}{2}\left[X_{1}(t+\right.$ $\left.\Delta \tau)-X_{2}(t)\right]$. The temporal evolution of these two variables is then given by

$$
\begin{aligned}
\dot{S}= & \frac{1}{2}[f(S+A)+f(S-A)] \\
& +\frac{1}{2}(L+K) C\left[S\left(t-\tau_{1}\right)+S\left(t-\tau_{2}\right)\right] \\
& +\frac{1}{2}(L+K) C\left[A\left(t-\tau_{1}\right)-A\left(t-\tau_{2}\right)\right], \\
\dot{A}= & \frac{1}{2}[f(S+A)-f(S-A)] \\
& +\frac{1}{2}(L-K) C\left[S\left(t-\tau_{1}\right)-S\left(t-\tau_{2}\right)\right] \\
& +\frac{1}{2}(L-K) C\left[A\left(t-\tau_{1}\right)+A\left(t-\tau_{2}\right)\right] .
\end{aligned}
$$

It is clear that a (time-shifted) synchronized solution $[A(t)=$ $0]$ only exists if the self-coupling and cross-coupling are equal $(L=K)$. This is in contrast to the case without delay mismatch (see Sec. II), where for any values of $K$ and $L$, a synchronized solution exists, although it may be unstable. Thus for the case with delay mismatch, we expect a strong dependence of the synchronization quality on the coupling mismatch $L-K$.

For $K=L$, the synchronized solution corresponds to one laser subject to two different feedbacks with the same strength $\frac{1}{2}(K+L)=K$ and respective delays $\tau_{1}$ and $\tau_{2}$. A linear stability analysis of Eq. (16), orthogonal to the synchronization manifold around $A(t)=0$, then leads to

$$
\dot{\delta A}(t)=D f(S) \delta A .
$$
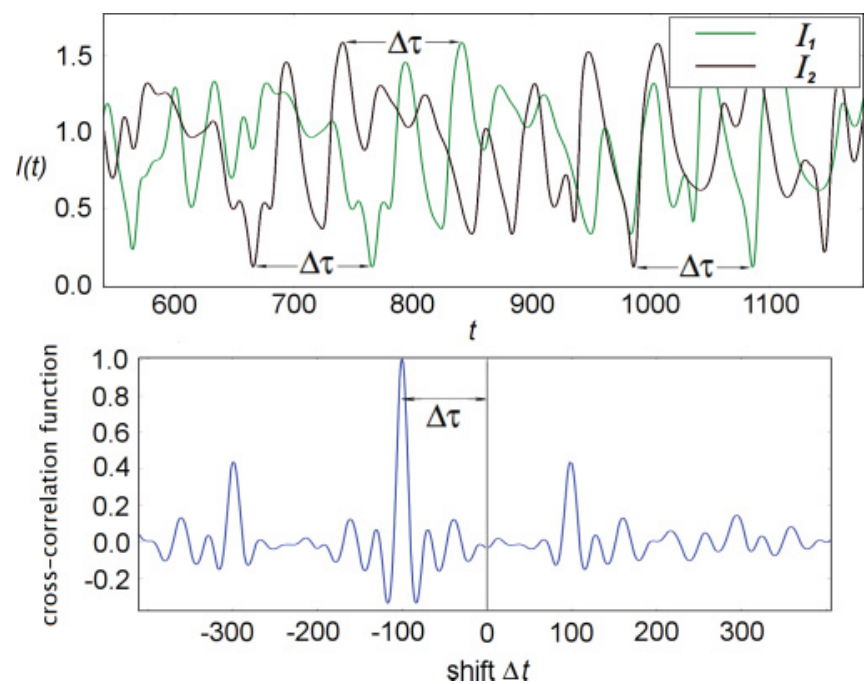

FIG. 7. (Color online) Dynamics and correlation of two delaycoupled lasers with a delay mismatch of $\Delta \tau=100$ in the coherence collapse regime. Upper panel: time series of laser output intensities $I_{1}(t)$ [green (dark gray) line] and $I_{2}(t)$ (black line). The time traces exhibit a time lag of $\Delta \tau$. For demonstration purposes, the noise was disregarded in this simulation. Lower panel: cross-correlation function of laser intensities. The time shift of the maximum correlation peak equals the delay mismatch: $\Delta t=-\Delta \tau$. Simulated with noise $\beta=10^{-5}$. Parameters for both simulations are $\tau=2000$, $\alpha=4.0, p=1.0, \mu=0.26, T=200$; all coupling strengths were identical $L=K=0.1$. 
Comparing the stability of the synchronization manifold without delay mismatch [Eq. (8)] for $L=K$ and with delay mismatch [Eq. (17)], we see that the variational equations are identical, and thus similar ECMs [i.e., solutions $S(t)$ with similar frequency and carrier density] of both systems will have comparable stability properties. Thus we can suspect that an asymmetrically placed mirror does not have a large effect on the synchronization properties of the system, provided that $L=K$. When simulating the system numerically, we indeed find identical synchronization of the two semiconductor lasers, with a time lag corresponding to the difference in propagation time between the lasers and the mirror, as shown in Fig. 7 (upper panel). Also, in the cross-correlation function, the main correlation peak is shifted by $\Delta \tau$ (see Fig. 7 , lower panel) as expected.

How does the peak height, i.e., the lag correlation, depend on the delay mismatch? In Fig. 8, the upper red curve depicts the height of the correlation peak as a function of $\Delta \tau$. For most of the allowed range $0 \leqslant \Delta \tau \leqslant \tau$, the correlation peak is large and the synchronization quality is high. The position of the mirror does not have a large effect on the synchronization level.

If we consider both a mismatch in the transmission and reflection of the mirror and a delay mismatch (Fig. 9), we find that the area around the diagonal $L=K$ synchronizes well, but the correlation breaks down even for a small mismatch in the coupling, in contrast to the case $\Delta \tau=0$ (Fig. 2). In other words, if the mirror is asymmetrically placed, the system is much more sensitive to a coupling mismatch, as was implied by Eq. (16) and discussed above.

\section{B. Dynamical regimes}

Although the delay mismatch does not affect the quality of the (lag) synchronization of the coupled lasers, it does have a significant effect on the dynamics in the synchronization

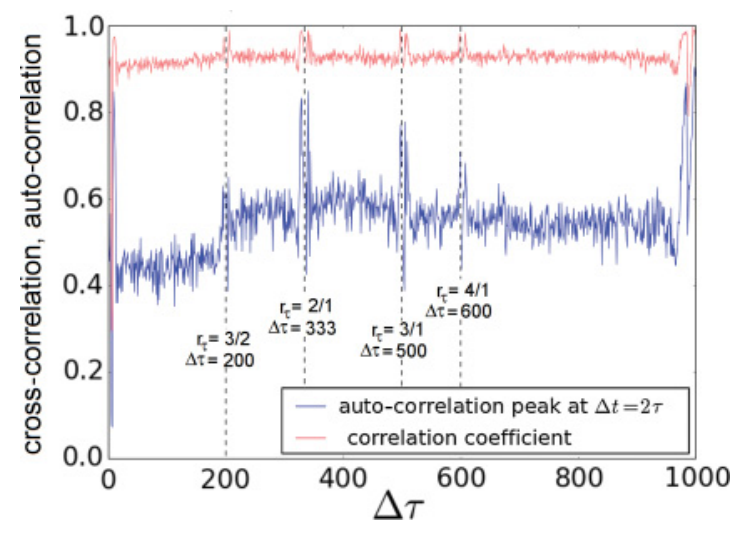

FIG. 8. (Color online) Cross-correlation $C(\Delta t)$ at shift $\Delta t=\Delta \tau$ of the output intensities of both lasers (upper red line), and the peak at $\Delta t=2 \tau$ of the autocorrelation function of one laser (lower blue line), versus the delay mismatch parameter $\Delta \tau$. The peaks coincide: higher regularity (peak in the autocorrelation) means stronger crosscorrelation (peak in the correlation coefficient). Parameters are $\tau=1000, \alpha=4.0, p=1.0, \mu=0.26, T=200, L=K=0.1$, $\beta=10^{-5}$.

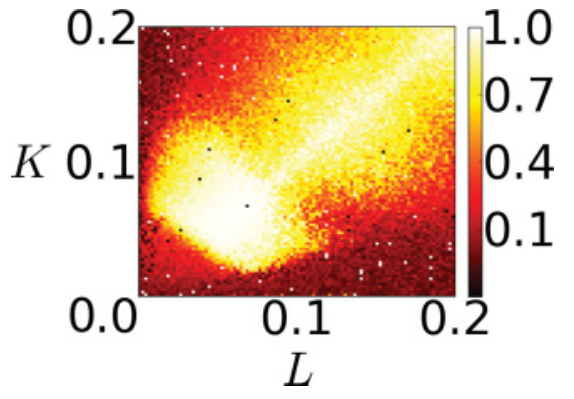

FIG. 9. (Color online) Cross-correlation $C(\Delta t)$ at shift $\Delta t=\Delta \tau$ of the field intensities of two coupled lasers in a configuration, according to Fig. 6, vs $L$ and $K$. In comparison with Fig. 2, the system is more sensitive to a mismatch of the coupling $L-K . \Delta \tau=$ 20. Other parameters are $\tau=1000, \mu=0.26, \alpha=4.0, p=1.0$, $T=200, \beta=10^{-5}$.

manifold itself. When synchronized (with a time lag), the two subsystems behave like one laser, subject to two equally strong feedbacks with different delays. This introduction of a second delay changes the alignment of the ECMs. The ECM ansatz $E(t)=A e^{i \omega t}, n(t)=n$ leads, after eliminating $A$, to a transcendental frequency equation and an equation for the carrier density,

$$
\begin{gathered}
\omega=-2 K \sqrt{1+\alpha^{2}} \sin (\arctan \alpha-\omega \tau) \cos (\omega \Delta \tau), \\
n=\frac{p \mu-2 K[\cos (\omega \tau) \cos (\omega \Delta \tau)]}{1+\mu} .
\end{gathered}
$$

The modes of this system are located inside the area of the ellipse that is the solution space for a laser subject to only one feedback with a strength of $2 K$. We note that if $r_{\tau}=\tau_{1} / \tau_{2} \in$ $\mathbb{Q}$, the modes lie on a closed curve, and irrational ratios $r_{\tau}$ result in a more complicated mode spectrum. Tronciu et al. [12], who investigated the corresponding case of lasers subject to feedback from an integrated double cavity, found chaotic behavior for lower feedback strengths than needed for the case of single feedback. The more complex mode alignment was postulated as a cause for a more complex dynamics. The complex alignment of the modes for $\Delta \tau \neq 0$ only depends on the delay mismatch, not on the delay time $\tau$. Since the dynamics is organized by the mode spectrum, it is strongly influenced by the changes in the mode structure.

For a small mismatch, the double delay setup exhibits a filtering effect similar to a Michelson interferometer [51], since the frequencies for which $\omega \Delta \tau \approx(2 m+1) \frac{\pi}{2}, m \in \mathbb{Z}$ are filtered out. This effect results in the formation of "mode islands." Close to simple rational ratios of $r_{\tau}$, we observe similar phenomena.

In Fig. 10, exemplary phase-space portraits are shown for different values of the delay mismatch $\Delta \tau$ for a fixed mean delay time $\tau=1000$. The areas where the transversely stable modes are located are similar for all delay mismatches $\Delta \tau$. Due to the different mode spectrum, the dynamics is significantly altered compared to the case $\Delta \tau=0$.

A semiconductor laser subject to two self-feedbacks with different strength and delay has been studied by Liu and Ohtsubo [52], who showed that one can stabilize the dynamics to fixed points or limit cycles. This effect was found strongest for unequal feedback strengths. Rogister et al. [53] showed 


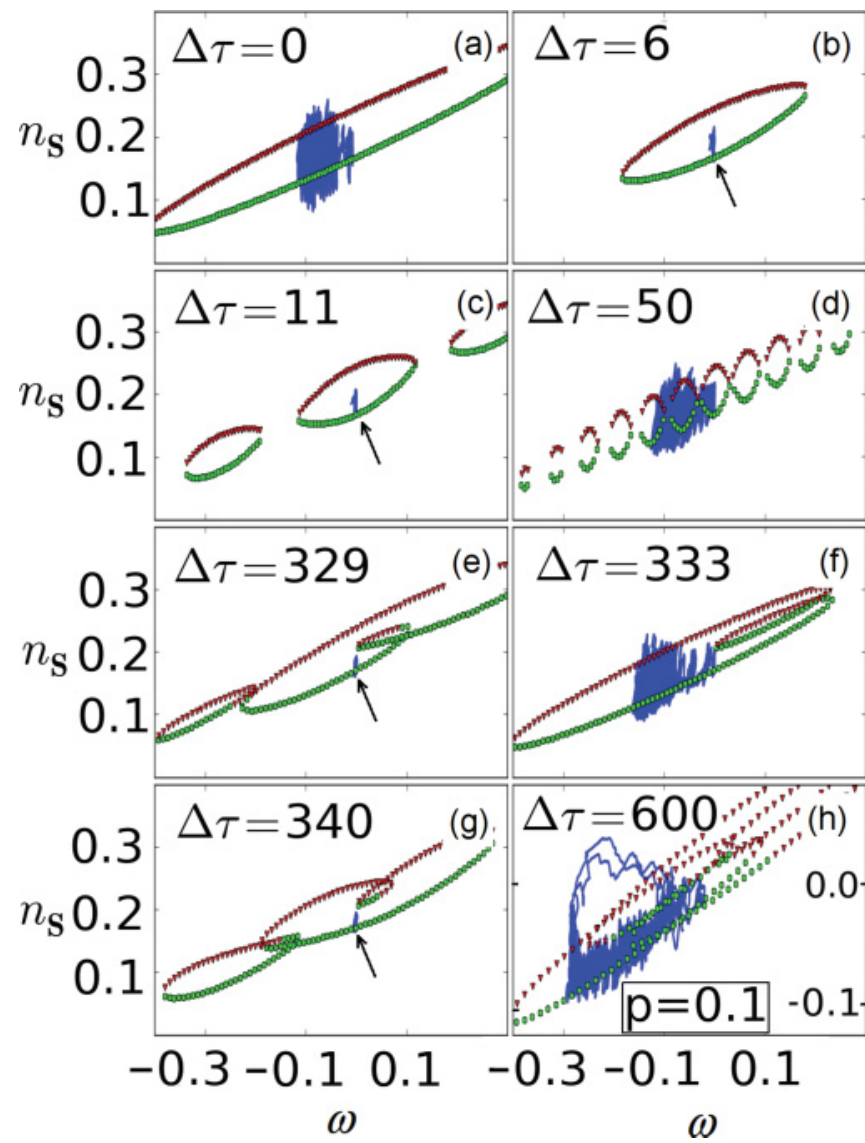

FIG. 10. (Color online) Dynamics of the symmetrized solution $\left(E_{S}, n_{S}\right)$ in the $\left(\omega, n_{S}\right)$ phase space for different values of the delay mismatch parameter $\Delta \tau$ after $t=5 \times 10^{4}$. For a definition of $\omega$ and $n_{S}$, see Fig. 3. The ECMs for a laser subject to two delayed feedbacks with different delay times are aligned inside the area of the mode ellipse for the case where the delays are equal. Green (light gray) circles are transversely stable modes; red (black) triangles are transversely unstable modes and antimodes. The itineracy is drawn in blue (dark gray) and marked with an arrow if stabilized. The mean delay time $\tau$ is fixed at $\tau=1000$. The other parameters are $\alpha=4$.0, $p=1.0$ (except lower right), $\mu=0.26, K=0.1$. Note that the lower right plot has a different $y$ scale than the others.

that chaotic dynamics and LFF can be suppressed for a single laser, subject to two different feedbacks, by suppressing the antimodes that are responsible for power dropouts [44]. This stabilization of the dynamics occurs mainly for a short second feedback. Increasing the second feedback strength from a low level to the magnitude of the first one results in a bifurcation cascade in the laser, leading to several dynamical regimes including stable behavior.

In a configuration of two lasers coupled via a semitransparent mirror, we observe similar changes in the dynamics. We calculated the secondary peak of the autocorrelation of each laser at $\Delta t=2 \tau$ to investigate the regularity of the time series. The height of the autocorrelation peak exhibits significant extrema (see Fig. 8, lower blue line) for certain delay mismatches. In particular, we find dips and peaks in the vicinity of simple rational values of $r_{\tau}$ and also around $r_{\tau}=1(\Delta \tau \approx 0)$ and $r_{\tau} \rightarrow \infty(\Delta \tau \approx \tau)$. At these points, naturally the cross correlation also exhibits dips and peaks

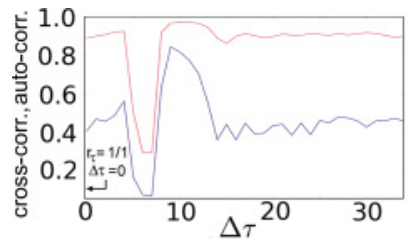

(a)

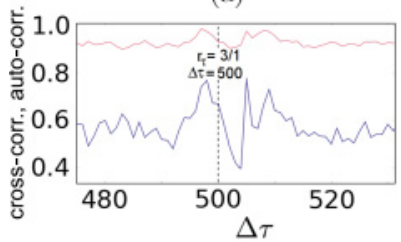

(c)

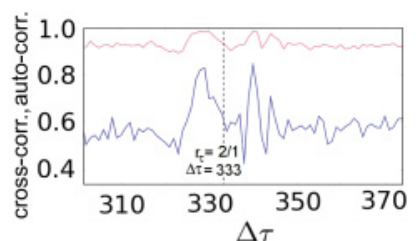

(b)

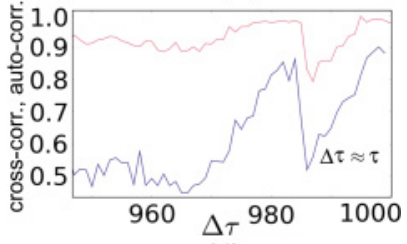

(d)
FIG. 11. (Color online) Cross-correlation coefficient (upper red lines), and auto-correlation at $\Delta t=2 \tau$ (lower blue lines), vs the delay mismatch parameter $\Delta \tau$ for rational ratios of the delay times $\tau_{1}$ and $\tau_{2}$. Magnifications of plot in Fig. 8. (a) $r_{\tau}=\frac{\tau_{1}}{\tau_{2}}=1 / 1$, (b) $r_{\tau}=2 / 1$, (c) $r_{\tau}=3 / 1$, (d) $\Delta \tau \approx \tau$. Parameters as in Fig. 8 .

(see Fig. 11, upper red line). These are primarily caused by a change in the underlying dynamical state of the system and not by a change of the synchronization quality. Lowered cross-correlation values have been tested to be caused by a small signal-to-noise ratio when the dynamics is stabilized to a fixed point. Delay mismatches that lead to a stabilization of the dynamics to a limit cycle result in a peak in the autocorrelation and cross correlation due to the coherent behavior.

To further investigate the changes in the laser dynamics for varying delay mismatch, we compute a bifurcation diagram. In the diagram, which is shown in Fig. 12, several intensity maxima and minima extracted from a time trace of each laser are plotted versus the delay mismatch $\Delta \tau$. The dynamics changes significantly for varying delay mismatch, when the delays are close to a simple ratio, e.g., $r_{\tau}=1 / 1,2 / 1,3 / 2, \ldots$ Especially

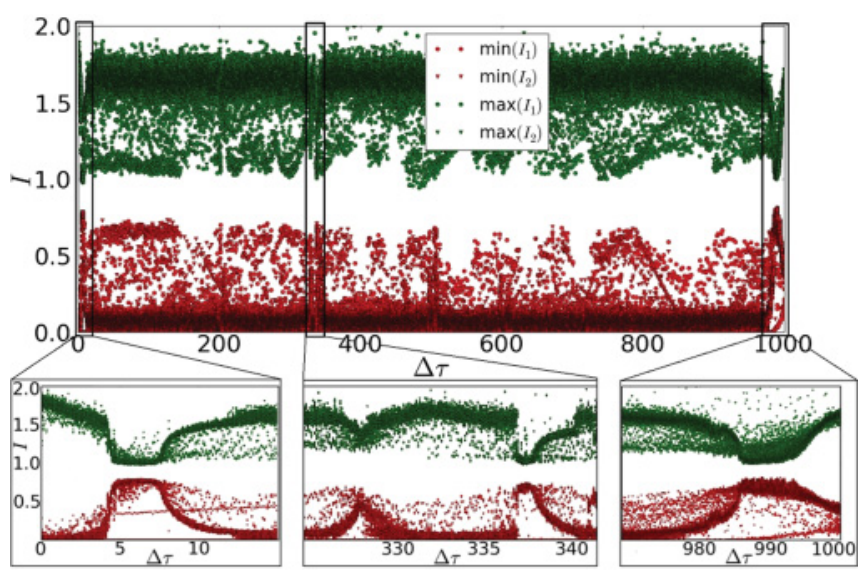

FIG. 12. (Color online) Bifurcation diagram of the intensity extrema of the two lasers vs the delay mismatch parameter $\Delta \tau$. Upper green points are maxima; lower red points are minima. Varying the delay mismatch results in radical changes in the dynamics, which is evident, e.g., by the changes in the variance of the intensity outputs. Magnifications are shown for a small delay mismatch $\Delta \tau<15$ (lower left), a delay times ratio of $r_{\tau} \approx 2 / 1$ (lower middle), and one very short delay $\tau_{1} \gg \tau_{2}$ (lower right). Other parameters are $\tau=1000$, $\alpha=4.0, \mu=0.26, p=1.0, T=200, \beta=10^{-5}$. 
for $\Delta \tau \approx 0$ (almost equal delay times) and $\Delta \tau \lesssim \tau$ (when one delay is very short), the variance of the intensity decreases dramatically. Also we notice a significant drop in variance at around $\Delta \tau=333$. This delay mismatch corresponds to a rational ratio of $r_{\tau}=2 / 1$ of the delay times. At these values of the delay mismatch, the dynamics settles on a stable periodic orbit.

The narrowest distances between maxima and minima indicate a stable single mode emission. For these cases, the autocorrelation and cross correlation show large peaks, however, this does not correspond to chaos synchronization.

The stabilization of the dynamics for several delay mismatches is illustrated in Fig. 10, which shows the laser dynamics projected onto the $(\omega, n)$ phase space. Close to the rational ratio $r_{\tau}=2 / 1(\Delta \tau \approx 333$ in this case), we see oscillations of the dynamics around a single mode [Figs. 10(e) and $10(\mathrm{~g})]$. The values for the delay mismatch correspond to those of the local peaks in the autocorrelation function in Fig. 11(b). At the exact rational ratio $\Delta \tau=333$, however, no such stabilization is observed [Fig. 10(f)]. For small delay mismatches [Figs. 10(b) and $10(\mathrm{c})$ ], the dynamics exhibits robust and fast stabilization to a single mode. This stabilizing property for a slightly misaligned semitransparent mirror is robust against the variation of several parameters, such as absolute delay time, coupling strength, and noise magnitude. Simulations over broad ranges of these respective parameters resulted in stabilization of the dynamics. With a strong enough nonlinear gain saturation, the dynamics and mode spectrum can be adjusted in such a way that high-level synchronization and the stabilization of the dynamics described above occur. (For more details on the effect of gain saturation, see the next section).

To explain the stabilization at small delay mismatches and at delay mismatches close to simple delay ratios $r_{\tau}$, we speculate as follows. Consider a laser subject to a delayed feedback from two feedback loops with delays $\tau+\Delta \tau$ and $\tau-\Delta \tau$. Let us assume that at $\Delta \tau=0$, the laser operates in a chaotic regime, where the chaos is induced by the feedback. Although the laser output is chaotic, there is, on average, a short-term phase correlation of the electric field, i.e., the peak at the origin of the autocorrelation function of the field is surrounded by local minima and maxima. The first local minimum and maximum is usually very pronounced because of a characteristic small oscillation period present in the chaotic signal. If we now change $\Delta \tau$ such that it corresponds to the first minimum, then, on average, the arriving feedback signals will destructively interfere, thus diminishing the amplitude and the fluctuations of the feedback signal, which may lead to more regular behavior of the laser. The above usually also holds for the choice of a small rational ratio of the delay times, i.e., $\frac{\Delta \tau}{\tau} \in \mathbb{Q}$. Of course, shifting $\Delta \tau$ will result in a different autocorrelation function, in particular in the case of successful stabilization. However, it can still be assumed that this kind of destructive interference mechanism can, in a self-consistent way, result in stabilization of the dynamics.

\section{NONLINEAR GAIN SATURATION}

For our analysis, we have used a Lang-Kobayashi-type model with a nonlinear gain function $G\left(E_{j}, n\right)$. A nonlinear

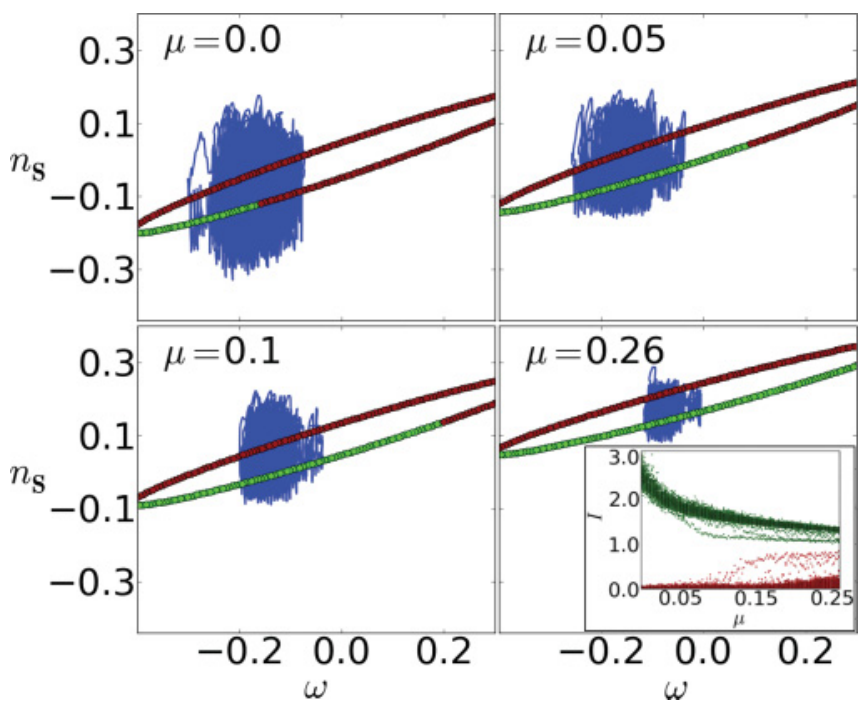

FIG. 13. (Color online) Effect of the nonlinear gain saturation on the alignment and transverse stability of the external cavity modes in the $\left(\omega, n_{S}\right)$ phase space; $p=1.0$. The larger the pump current value is, the more pronounced is the effect. Red (black) circles: transversely unstable antimodes. Green (light gray) circles: transversely stable modes. The dynamics is shown in blue (dark gray). The other parameters are $\tau=1000, \Delta \tau=0, \alpha=4.0, T=200$, $K=0.1, \beta=10^{-5}$. Inset: bifurcation diagram with varying $\mu$. Upper green points: maxima of intensities; lower red points: minima of intensities.

gain, which saturates for high intensities, is often used to account for nonlinear deviations of the characteristics of the optical power versus injection current far above the threshold.

The nonlinear gain saturation is a phenomenologically introduced term that is motivated by nonlinear effects in the semiconductor gain medium, like spectral hole burning and carrier heating. A linear gain theory cannot account for those phenomena.

With increasing pump current, the nonlinear gain saturation becomes more relevant and has an increasing effect on the dynamics of the lasers, e.g., on the precise position in phase space [see Eq. (19)] and the transverse stability of the external cavity modes.

When operating in the LFF regime, the effect of nonlinear gain saturation can often be neglected. In the coherence collapse (CC) regime, however, the high power means that gain saturation plays an increasing role. This effect is illustrated in Fig. 13. Increasing the nonlinear gain saturation $\mu$ leads to a smaller maximum field intensity and a decreased variance of the intensity and carrier density. The inset in Fig. 13 shows this effect via a distribution of intensity extrema for varying $\mu$.

In the coupled laser system, the number of transversely stable modes increases with increasing $\mu$ and the modes are shifted so that critical events with antimodes and desynchronization are less likely - the synchronized state is more stable. However, the dynamics is also less complex.

With a model that omits the nonlinear gain $(\mu=0)$, all modes in the vicinity of the minimum gain mode (around $\omega=0$ ) are transversely unstable above a critical pump current. Without gain saturation, the lasers experience many 


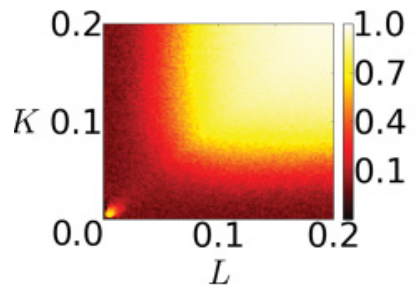

(a)

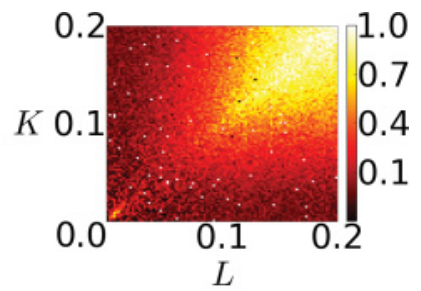

(b)
FIG. 14. (Color online) Cross-correlation of the field intensities vs $K$ and $L$ with and without delay mismatch. (a) Symmetric delays: $\Delta \tau=0$, zero-lag synchronization; (b) with delay mismatch $\Delta \tau=$ 20 , cross-correlation at $\Delta t=\Delta \tau$. The nonlinear gain saturation is ignored for these simulations. Parameters: $\mu=0, p=1.0, \alpha=4.0$, $T=200, \tau=1000$, and noise $\beta=10^{-5}$.

desynchronization events or do not synchronize at all. The $L-K$ plots in Fig. 14 show therefore significantly smaller areas of high synchronization for $\mu=0$ than the corresponding plots for $\mu=0.26$ (Figs. 2 and 9).

\section{CONCLUSIONS}

We have numerically and analytically investigated the dynamical and synchronization properties of a system of two delay-coupled semiconductor lasers in different coupling schemes. Using a model for a setup of two lasers coupled via a semitransparent mirror, we have studied the influence of a mismatch between the transmission and reflection of the mirror, i.e., a mismatch between coupling and feedback strength, as well as a delay mismatch corresponding to a misalignment of the mirror from the middle.

We showed that a coupling mismatch deteriorates the stability of the synchronized solution, but does not change the synchronized dynamics. In both the LFF regime and the $\mathrm{CC}$ regime, a larger mismatch results in longer bubbling events, since less modes are transversely stable. However, we still observe high-level synchronization if the mismatch $|L-K|$ remains small. The cross-correlation (at zero lag) of the two lasers does not depend on the sign of the mismatch between coupling and feedback $L-K$, but only on the absolute value.

The inclusion of a saturable nonlinear gain leads not only to a reduction of complexity in the time series but also to a broader domain of high synchronization quality, since it increases the transverse stability of the ECMs.

Our analysis has shown that the synchronization properties of a configuration with a semitransparent mirror are the same as those of a drive-response configuration, at least on the level of the ECMs. An open-loop configuration synchronizes best; the transverse stability of the modes is the same as in a configuration without coupling mismatch. Adding self-feedback to the receiver has the same effect on stability as introducing a coupling mismatch in the relay setup.

If there is a delay mismatch, we found that the lasers can synchronize with a nonzero lag. The time traces then exhibit a relative time shift proportional to the misalignment of the mirror. The stability of the time-shifted identical synchronization of the lasers is not much affected by the value of the delay mismatch. The dynamics, however, undergoes drastic changes for varying $\Delta \tau$, as the alignment of the external cavity modes is altered compared to symmetric delays.

Especially for very small mismatch, for rational ratios of the respective delay times, and if the second delay is very short compared to the first one, the chaotic dynamics can be suppressed and stabilized toward either single mode output or periodic behavior. These qualitative features do not depend on the delay time, but merely on the delay mismatch and relative ratios of delay times. They were found robust for broad ranges of the coupling strengths (i.e., the transmission and reflection of the relay mirror) and even for high noise levels. We conjecture that they are caused by destructive interference.

We also found that a delay mismatch makes the system much more sensitive to a coupling mismatch. The correlation between the two laser outputs decreases much more rapidly with increasing coupling mismatch than for the case of symmetric delays.

Our results are of high practical value for future (experimental) investigations considering delay-coupled lasers. The ability to target specific stability regions via mismatch adjustments can, for example, be helpful for chaos communication systems or random number generation. In addition, due to the broad interest in synchronization phenomena throughout the scientific community, our results concern not only the immediate applications to chaotic communication, but may also be relevant to scientists in related fields such as computational neuroscience, engineering, and biology. The presented analytical results can be transferred to other delay-coupled systems with corresponding network topology and therefore could find applications, e.g., in electro-optic or neuronal systems.

\section{ACKNOWLEDGMENTS}

O.D. acknowledges the Research Foundation Flanders (FWO-Vlaanderen) for support. This research was partially supported by MICINN (Spain) under project DeCoDicA (Project No. TEC2009-14101), by DFG (Germany) in the framework of SFB 910, by the Interuniversity Attraction Poles program of the Belgian Science Policy Office, under Grant No. IAP VI-10 (photonics@be), and by the project PHOCUS. The project PHOCUS, in turn, acknowledges the financial support of the Future and Emerging Technologies (FET) program within the Seventh Framework Programme for Research of the European Commission, under FET-Open Grant No. 240763.

\section{APPENDIX : STABILITY CALCULATION FOR CAVITY MODES}

An external cavity mode (ECM) of the Lang-Kobayashi equations is a steady-state solution of the form $E_{j}=A e^{i \omega t}$ and $n_{j}=n$. To calculate its (transverse) stability, we make the transformation $E_{j} \rightarrow E e^{-i \omega t}$ such that the ECM becomes a steady state, and split the electric field into a real and imaginary part $E=A=x+i y$. With this, we calculate the Jacobian $D f(S)$ with trajectory $S$ corresponding to the transformed ECM solution. We then determine the eigenvalues of the 
linearized right-hand side of Eq. (8),

$\operatorname{det}\left[\begin{array}{ccc}\frac{1}{2}\left(G-1-2 x^{2} \frac{\partial G}{\partial I}\right)-\lambda & \omega-\frac{\alpha}{2}\left(G-1-{ }^{2} y^{2} \frac{\partial G}{\partial I}\right) & \frac{1}{2} \frac{\partial G}{\partial n}(x-\alpha y) \\ -\omega+\frac{\alpha}{2}\left(G-1-{ }^{2} y^{2} \frac{\partial G}{\partial I}\right) & \frac{1}{2}\left(G-1-2 x^{2} \frac{\partial G}{\partial I}\right)-\lambda & \frac{1}{2} \frac{\partial G}{\partial n}(\alpha x+y) \\ -\frac{2 x}{T}\left(G+I \frac{\partial G}{\partial I}\right) & -\frac{2 y}{T}\left(G+I \frac{\partial G}{\partial I}\right) & -\frac{1}{T}\left(1+I \frac{\partial G}{\partial n}\right)-\lambda\end{array}\right]-(L-K) e^{-\lambda \tau}\left(\begin{array}{ccc}1 & 0 & 0 \\ 0 & 1 & 0 \\ 0 & 0 & 0\end{array}\right)=0$,

where $I$ represents the intensity of the laser, $I=|A|^{2}=x^{2}+y^{2}$.

If all of the resulting eigenvalues have negative real parts, it indicates that the ECM is transversely stable: the magnitude of the small perturbation $\delta(t)$ in Eq. (8) decreases over time. However, if at least one eigenvalue has a positive real part, it means the ECM in question is transversely unstable.

[1] A. S. Pikovsky, M. G. Rosenblum, and J. Kurths, Synchronization, A Universal Concept in Nonlinear Sciences (Cambridge University Press, Cambridge, 2001).

[2] S. Boccaletti, J. Kurths, G. Osipov, D. L. Valladares, and C. S. Zhou, Phys. Rep. 366, 1 (2002).

[3] Handbook of Chaos Control, edited by E. Schöll and H. G. Schuster (Wiley-VCH, Weinheim, 2008).

[4] W. Singer, Neuron 24, 49 (1999).

[5] W. Singer, Nature (London) 397, 391 (1999).

[6] L. M. Pecora and T. L. Carroll, Phys. Rev. Lett. 64, 821 (1990).

[7] L. M. Pecora and T. L. Carroll, Phys. Rev. A 44, 2374 (1991).

[8] L. M. Pecora, T. L. Carroll, G. A. Johnson, D. J. Mar, and J. F. Heagy, Chaos 7, 520 (1997).

[9] K. M. Cuomo and A. V. Oppenheim, Phys. Rev. Lett. 71, 65 (1993).

[10] G. D. VanWiggeren and R. Roy, Phys. Rev. Lett. 81, 3547 (1998).

[11] A. Sanchez-Diaz, C. R. Mirasso, P. Colet, and P. GraciaFernandez, IEEE J. Quantum Electron. 35, 292 (1999).

[12] V. Z. Tronciu, C. R. Mirasso, and P. Colet, J. Phys. B 41, 155401 (2008).

[13] M. C. Soriano, P. Colet, and C. R. Mirasso, IEEE Photon. Technol. Lett. 21, 426 (2009).

[14] A. Argyris, D. Syvridis, L. Larger, V. Annovazzi-Lodi, P. Colet, I. Fischer, J. García-Ojalvo, C. R. Mirasso, L. Pesquera, and K. A. Shore, Nature (London) 438, 343 (2005).

[15] I. Fischer, O. Hess, W. Elsäßer, and E. O. Göbel, Phys. Rev. Lett. 73, 2188 (1994).

[16] V. Ahlers, U. Parlitz, and W. Lauterborn, Phys. Rev. E 58, 7208 (1998).

[17] W. Just, A. Pelster, M. Schanz, and E. Schöll, Phil. Trans. R. Soc. A 368, 303 (2010).

[18] C.-U. Choe, T. Dahms, P. Hövel, and E. Schöll, Phys. Rev. E 81, 025205(R) (2010).

[19] V. Flunkert, S. Yanchuk, T. Dahms, and E. Schöll, Phys. Rev. Lett. 105, 254101 (2010).

[20] O. D'Huys, R. Vicente, J. Danckaert, and I. Fischer, Chaos 20, 043127 (2010).

[21] W. Kinzel, A. Englert, G. Reents, M. Zigzag, and I. Kanter, Phys. Rev. E 79, 056207 (2009).

[22] J. K. White, M. Matus, and J. V. Moloney, Phys. Rev. E 65, 036229 (2002)

[23] T. Heil, I. Fischer, W. Elsäßer, J. Mulet, and C. R. Mirasso, Phys. Rev. Lett. 86, 795 (2001).
[24] J. Mulet, C. R. Mirasso, T. Heil, and I. Fischer, J. Opt. B6, 97 (2004).

[25] E. A. Rogers-Dakin, J. García-Ojalvo, D. J. DeShazer, and R. Roy, Phys. Rev. E 73, 045201 (2006).

[26] L. B. Shaw, I. B. Schwartz, E. A. Rogers, and R. Roy, Chaos 16, 015111 (2006)

[27] E. Klein, N. Gross, M. Rosenbluh, W. Kinzel, L. Khaykovich, and I. Kanter, Phys. Rev. E 73, 066214 (2006).

[28] R. Vicente, C. R. Mirasso, and I. Fischer, Opt. Lett. 32, 403 (2007).

[29] A. S. Landsman, L. B. Shaw, and I. B. Schwartz, in Recent Advances in Laser Dynamics: Control and Synchronization, edited by A. N. Pisarchik (Research Signpost, Kerala, India 2007), p. 359.

[30] I. B. Schwartz and L. B. Shaw, Phys. Rev. E 75, 046207 (2007).

[31] M. Peil, L. Larger, and I. Fischer, Phys. Rev. E 76, 045201 (2007).

[32] R. Vicente, G. Pipa, I. Fischer, and C. R. Mirasso, in Artificial Neural Networks ICANN 2007, Lecture Notes in Computer Science Vol. 4668 (Springer, Berlin/Heidelberg, 2007), pp. 904-913.

[33] R. Vicente, L. L. Gollo, C. R. Mirasso, I. Fischer, and P. Gordon, Proc. Natl. Acad. Sci. USA 105, 17157 (2008).

[34] R. Vicente, L. L. Gollo, C. R. Mirasso, I. Fischer, and G. Pipa, in Coherent Behavior in Neuronal Networks, edited by K. Josic, J. Rubin, M. A. Matias, and R. Romo , Computational Neuroscience Vol. 3 (Springer, Berlin/Heidelberg, 2009), pp. 143-167.

[35] J. Ohtsubo, Opt. Rev. 6, 1 (1999).

[36] A. Murakami and J. Ohtsubo, Phys. Rev. A 65, 033826 (2002).

[37] A. Locquet, C. Masoller, and C. R. Mirasso, Phys. Rev. E 65, 56205 (2002).

[38] Y. Nagai and Y. C. Lai, Phys. Rev. E 56, 4031 (1997).

[39] P. Ashwin, J. Buescu, and I. Stewart, Phys. Lett. A 193, 126 (1994).

[40] V. Flunkert, O. D’Huys, J. Danckaert, I. Fischer, and E. Schöll, Phys. Rev. E 79, 065201 (R) (2009).

[41] V. Rottschäfer and B. Krauskopf, Int. J. Bifurcation Chaos 17, 1575 (2007).

[42] H. Erzgräber, B. Krauskopf, and D. Lenstra, SIAM J. Appl. Dyn. Syst. 5, 30 (2006). 
[43] R. Lang and K. Kobayashi, IEEE J. Quantum Electron. 16, 347 (1980).

[44] T. Sano, Phys. Rev. A 50, 2719 (1994).

[45] J. Mørk, B. Tromborg, and P. L. Christiansen, IEEE J. Quantum Electron. 24, 123 (1988).

[46] S. Yanchuk and M. Wolfrum, in Proceedings of the Fifth EUROMECH Nonlinear Dynamics Conference ENOC-2005, Eindhoven, 2005, edited by D. H. van Campen, M. D. Lazurko, and W. P. J. M. van den Oever (Eindhoven University of Technology, Eindhoven, Netherlands, 2005), pp. 2069-2073.

[47] C. R. Mirasso, P. Colet, and P. Garcia Fernandez, IEEE Photon. Technol. Lett. 8, 299 (1996).
[48] I. Fischer, Y. Liu, and P. Davis, Phys. Rev. A 62, 011801 (2000).

[49] R. Vicente, T. Pérez, and C. R. Mirasso, IEEE J. Quantum Electron. 38, 1197 (2002).

[50] T. Heil, J. Mulet, I. Fischer, C. R. Mirasso, M. Peil, P. Colet, and W. Elsäßer, IEEE J. Quantum Electron. 38, 1162 (2002).

[51] S. E. Würtenberger, Master's thesis, Technische Universität Darmstadt, 2000.

[52] Y. Liu and J. Ohtsubo, IEEE J. Quantum Electron. 33, 1163 (1997).

[53] F. Rogister, P. Mégret, O. Deparis, M. Blondel, and T. Erneux, Opt. Lett. 24, 1218 (1999). 